\title{
ESPECTROSCOPIA OPTOGALVÂNICA: ALGUNS ASPECTOS RELEVANTES
}

Robson Valentim Pereira*

Instituto de Química de São Carlos, Universidade de São Paulo, CP 780, 13560-970 São Carlos - SP, Brasil

Francisco B. T. Pessine

Instituto de Química, Universidade Estadual de Campinas, CP 6154, 13084-971 Campinas - SP, Brasil

Recebido em 29/6/05; aceito em 6/1/06; publicado na web em 11/8/06

\begin{abstract}
OPTOGALVANIC SPECTROSCOPY: SOME RELEVANTS ASPECTS. In this work we present the optogalvanic effect in ionized gases in an historical perspective. This effect was observed for the first time by Foote and Mohler in 1925, and explained by Penning in 1928 for mixtures (Ne-Ar) and pure gases (or vapours) in 1937. Also, we show some aspects of the contributions of Romenian authors for the development of optogalvanic spectroscopy, which was used as a new technique only after 1964.
\end{abstract}

Keywords: optogalvanic effect; Romenian works; spectroscopy.

\section{INTRODUÇÃO}

Quando um gás ou o vapor de uma substância é ionizado mediante uma descarga elétrica efetuada entre dois eletrodos, através de radiação de microondas ou de rádio freqüência, várias espécies podem ser produzidas, como íons (atômicos, moleculares ou radicalares), átomos e/ou moléculas excitadas, radicais livres, etc. Ao iluminar uma amostra assim produzida com radiação ressonante à de absorção eletrônica da espécie formada, ocorrem alterações em suas propriedades elétricas. Este fenômeno é conhecido como efeito optogalvânico, o qual constitui a base da espectroscopia optogalvânica ${ }^{1,2}$, atualmente uma das principais ferramentas espectroscópicas, com aplicações nas áreas de Física, Química, Biologia e Engenharia. Entretanto, de modo geral, observa-se que ela é raramente utilizada por químicos embora apresente várias vantagens quando comparada com outras técnicas espectroscópicas, como fluorescência induzida por lasers, absorção atômica ou mesmo espectrometria de massa.

A Figura 1 mostra um esquema experimental típico empregado nos trabalhos com espectroscopia optogalvânica.

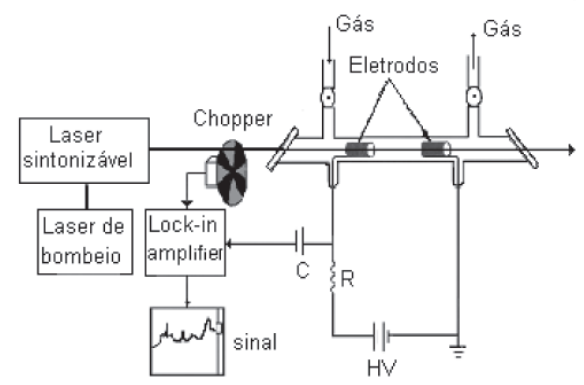

Figura 1. Arranjo de um sistema experimental usado em espectroscopia optogalvânica

A Figura 1 mostra uma fonte de luz, constituída de um laser sintonizável (laser de corante, $\mathrm{Ti}_{\mathrm{i}} \mathrm{Al}_{2} \mathrm{O}_{3}$ ), bombeado por outro laser

*e-mail: rpereira@iqsc.usp.br (geralmente $\mathrm{Nd}$ :YAG, $\mathrm{N}_{2}$, diodo, excímero). A luz é direcionada à região da descarga (produzida pelos eletrodos) mostrados na Figura 1 e, quando esta radiação for ressonante a uma transição eletrônica de uma espécie presente no plasma, esta absorve a radiação. A excitação das espécies presentes na descarga de um estado eletrônico para outro altera a impedância da descarga entre os eletrodos, constituindo o sinal optogalvânico.

Ao contrário do que se encontra geralmente mencionado na literatura, o efeito optogalvânico foi observado pela primeira vez por Foote e Mohler ${ }^{3,4}$, em 1925, empregando vapor de césio. Em 1928 Penning 5 , em um artigo denominado "Demonstração de um novo efeito fotoelétrico", expôs a importância de átomos metaestáveis na condutividade elétrica de uma descarga, fornecendo a primeira interpretação física sobre o efeito optogalvânico. Posteriormente, outros cientistas estudaram o fenômeno responsável por este efeito, como Kenty ${ }^{6}$, Meissner e Miller ${ }^{7}$, Phelps e Molnar ${ }^{8}$. Variações na impedância em descargas elétricas também foram observadas em lasers à gás (como o de He-Ne) por Garscaden et al. ${ }^{9}$, em descargas de Xe por Freiberg et al. ${ }^{10}$, em lasers de $\mathrm{CO}_{2}$ por Carswell et al. ${ }^{11}$. Contudo, o uso sistemático deste efeito como uma nova metodologia espectroscópica começou apenas em 1964 com trabalhos de pesquisadores romenos sobre descargas de baixa potência, efetuadas com radiação de rádio frequência, em vapores de metais alcalinos, especialmente césio, aplicando, posteriormente, a mesma metodologia a gases e vapores de outros compostos.

Neste artigo serão abordados os fenômenos associados ao efeito optogalvânico e alguns trabalhos de pesquisadores romenos pioneiros no desenvolvimento desta técnica.

\section{OBJETIVO}

Já há algum tempo a espectroscopia optogalvânica ocupa lugar de destaque dentre as principais técnicas de absorção. Porém, equivocadamente, vários artigos ${ }^{1,2,12-20}$ atribuem a Penning a descoberta do efeito optogalvânico, que constitui a essência desta técnica. Estes artigos citam o trabalho publicado por esse autor ${ }^{5}$, o qual explica o efeito optogalvânico somente para a mistura de gases (Ne-Ar), sendo necessário apresentar o trabalho de Penning envolvendo descargas de gases (ou vapor) puros, pois o efeito optogalvânico e, conseqüentemente, a espectroscopia optogalvânica não é aplicada somente a misturas de gases. 
De modo geral, muitos artigos dentre os citados acima e até mais recentes ${ }^{21}$ consideram o trabalho de Green et al. ${ }^{22}$, em 1976, como um marco decisivo no desenvolvimento da espectroscopia optogalvânica. No entanto, é preciso considerar os trabalhos pioneiros dos pesquisadores romenos ${ }^{23-34}$ que desenvolveram e aplicaram sistematicamente este tipo de espectroscopia pela primeira vez em 1964, e que continuaram a empregá-la, juntamente com Collins et $a .^{27-34}$. Esta técnica, denominada por eles de espectroscopia de impedância óptica, juntamente com o trabalho de Green et al..$^{22}$, passou a ser conhecida como espectroscopia optogalvânica.

Espera-se neste trabalho esclarecer a descoberta do efeito optogalvânico, apresentando os trabalhos de Penning em misturas de gases e em gases ou vapores puros, assim como citar a importância dos trabalhos romenos no desenvolvimento desta importante ferramenta espectroscópica.

\section{O EFEITO OPTOGALVÂNICO}

O efeito fotoelétrico em gases ionizados foi demonstrado pela primeira vez por Foote e Mohler ${ }^{3,4}$ em vapor de césio, utilizando como detector um filamento de tungstênio desenvolvido por Langmuir e Kingdon ${ }^{35,36}$.

Foote e Mohler iluminaram a região de uma descarga elétrica produzida em vapor de césio com luz gerada em uma lâmpada de Mazda com $600 \mathrm{~W}$ de potência, observando uma variação na corrente da descarga, como mostra a Figura 2.

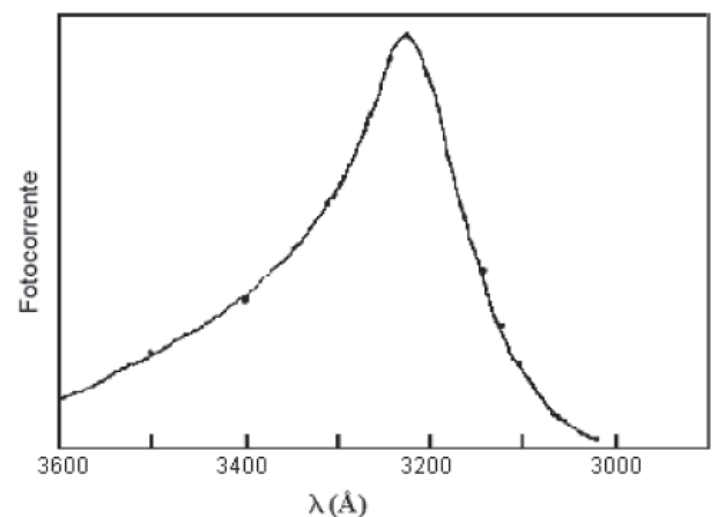

Figura 2. Variação espectral da corrente elétrica de uma descarga em vapor de césio.

Segundo esses autores, o máximo da curva (que ocorre em 3231 Å) apresenta evidências de que a radiação absorvida é gasta na ionização das espécies excitadas existentes na descarga, como descrito por Einstein na equação sobre o efeito fotoelétrico. As variações da corrente elétrica que ocorrem em outros valores do comprimento de onda $\lambda$ corresponderiam a colisões entre átomos excitados, que contribuiriam para essa corrente.

Em 1928, Penning 5 iluminou um tubo contendo neônio e uma pequena $(0,001 \%)$ quantidade de argônio, com luz proveniente de outro tubo de neônio e observou um aumento (de cerca de $50 \mathrm{~V}$ ) na tensão entre os eletrodos (diminuição da corrente), interpretando este resultado de acordo com sua teoria de ionização dos átomos metaestáveis de Ne por colisões com átomos de argônio. De acordo com ele este aumento era devido a uma diminuição do número de átomos metaestáveis provocada pela irradiação com a luz emitida pelo mesmo gás. A Figura 3 mostra os processos envolvidos em uma mistura Ne-Ar.

Nesta Figura apenas os dois níveis 1s mais importantes são representados $\left(1 \mathrm{~s}_{4}\right.$ e o metaestável $\left.1 \mathrm{~s}_{5}\right)$ e os níveis $2 \mathrm{p}$ todos represen-

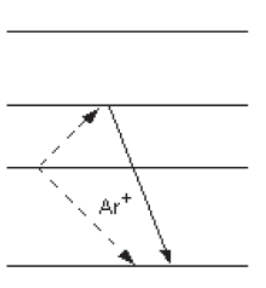

a)

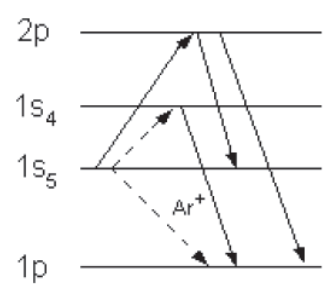

b)
Figura 3. Processos de excitação envolvidos em uma mistura de Ne-Ar, sem irradiação (a) e com irradiação (b)

tados por uma linha. As transições radioativas são representadas por linhas cheias, enquanto as não radioativas por linhas tracejadas.

Quando o tubo de descarga não é iluminado, os átomos metaestáveis de Ne que estão no nível $1 \mathrm{~s}_{5}$ podem ser destruídos de duas maneiras: por colisões com outros átomos de neônio, podem ser levados ao estado $1 \mathrm{~s}_{4}$, do qual retornam ao nível fundamental $1 \mathrm{p}$ por emissão em $7360 \AA$, e por colisões com átomos de $\mathrm{Ar}$, sendo estes últimos ionizados neste processo.

Ao iluminar o tubo de descarga com radiação gerada em um segundo tubo de neônio, os átomos metaestáveis de $\mathrm{Ne}\left(1 \mathrm{~s}_{5}\right)$ podem ser levados ao nível $2 \mathrm{p}$, do qual eles retornam (via nível $1 \mathrm{~s}_{4}$ ) para o estado fundamental (Figura 3b). Assim, com irradiação, poucos átomos de argônio são ionizados por colisão com os átomos metaestáveis de Ne e o potencial da descarga aumenta.

Mais detalhes sobre esta teoria foram publicados posteriormente por ele em outros artigos ${ }^{37,38}$. Segundo Penning, a diminuição do potencial em uma descarga era devida à ionização por átomos metaestáveis e, para este processo ocorrer, a condição seguinte deveria ser obedecida:

$\mathrm{V}_{\mathrm{i}}^{\prime}<\mathrm{V}_{\text {met }}$

onde $\mathrm{V}_{\mathrm{i}}$ 'é o potencial de ionização dos átomos da impureza (argônio, presente em menor concentração) na descarga e $\mathrm{V}_{\text {met }}$ é o potencial dos átomos metaestáveis do gás principal.

Segundo Penning ${ }^{37-39}$, o potencial de uma descarga, contendo traços de outro gás, poderia ser novamente aumentado com a diminuição da concentração de átomos metaestáveis (essenciais para a manutenção da corrente na descarga). Ele mostrou que isto poderia ser feito de duas maneiras: adicionando um terceiro gás à mistura ou iluminando a descarga, como já descrito.

No primeiro caso, Penning mostrou que a adição de $\mathrm{He}$ ou $\mathrm{N}_{2}$ em uma mistura de $\mathrm{Ar}$ e $\mathrm{Hg}$ provocava um aumento da tensão. Adicionando $1 \%$ de $\mathrm{N}_{2}$ o potencial da descarga passou de 220 para 520 V. Por outro lado, a adição de $\mathrm{N}_{2}$ em uma descarga de Ar puro mostrou somente um leve aumento na tensão.

Em 1937, Penning ${ }^{40}$ demonstrou que um tubo contendo gás (ou vapor) puro ao ser iluminado com luz produzida pelo mesmo gás (ou vapor) também provocava variações na impedância de uma descarga. Segundo ele, um átomo (molécula) de um gás ou vapor é excitado inicialmente a um estado metaestável através de colisão com um elétron e, então, é ionizado por um segundo elétron. Ao iluminar a descarga, as espécies metaestáveis podem ser levadas a estados não metaestáveis de alta energia e de tempo de vida muito menor que o do estado metaestável. Assim, a probabilidade delas serem ionizadas por elétrons é extremamente pequena, e eles não contribuem para a corrente da descarga, como acontece com átomos existentes nos níveis metaestáveis. Portanto, a tensão entre os eletrodos é maior que no caso onde a amostra não é iluminada.

Como já descrito na Introdução deste trabalho, variações na 
condutividade elétrica de uma descarga também foram encontradas em descargas de lasers à gás, e trabalhos semelhantes aos de Penning foram realizados por Kenty ${ }^{6}$ que observou variações significativas da voltagem no tubo de descarga, quando este foi iluminado com luz produzida em uma lâmpada de $\mathrm{Hg}$, e que a adição de $\mathrm{N}_{2}$ provocava o aumento nessa tensão. Meissner e Miller ${ }^{7}$ também observaram este efeito trabalhando com gases nobres, demostrando que realmente em uma descarga elétrica os átomos metaestáveis desempenham papel importante como fonte de ionização secundária, via colisões, como já havia sido sugerido por Penning.

\section{TRABALHOS PIONEIROS}

O uso sistemático do efeito optogalvânico como um novo princípio para espectroscopia de absorção começou em 1964 com trabalhos de pesquisadores romenos ${ }^{23-25,27,31}$ envolvendo descargas de baixa potência em vapores de metais alcalinos, onde as transições associadas às séries de Lyman, Balmer, Paschen e Pfund foram resolvidas, sendo usado um diodo termiônico como detector, o qual mostrou alta sensibilidade para detecção dos íons formados na descarga. Assim Badareu et al. ${ }^{23}$ adicionaram mercúrio sobre vapor de césio, sendo observado um aumento na eficiência de ionização destes. Segundo os autores, isto é devido à ionização dos átomos de césio por colisões de segundo tipo com átomos excitados de mercúrio (efeito Penning), como mostra a Equação 1

$\mathrm{Hg}^{*}+\mathrm{Cs} \rightarrow \mathrm{Hg}+\mathrm{Cs}^{+}+\mathrm{e}$

onde $\mathrm{Hg}^{*}$ representa mercúrio no estado excitado.

Em um trabalho posterior ${ }^{24}$ muito parecido com o descrito anteriormente, os autores adicionaram cádmio ao vapor de césio, já que a adição de mercúrio provocava a formação de amálgamas diminuindo, assim, a pressão parcial da mistura. Novamente verificou-se que o cádmio desempenhava um papel fundamental na ionização dos átomos de césio, conforme indicado na Equação 2

$\mathrm{Cd}^{*}+\mathrm{Cs} \rightarrow \mathrm{Cd}+\mathrm{Cs}^{+}+\mathrm{e}$

onde $\mathrm{Cd}^{*}$ indica cádmio no estado excitado.

Esses artigos ${ }^{23-25,27,31}$ também relatam transições envolvendo a série principal, sendo resolvidas as que ocorrem entre $n=9$ e $n=19$; $n=9$ e $n=42$ e $n=9$ e $n=40$, o que permitiu a obtenção de novos dados a respeito do mecanismo de fotoionização do vapor de césio, e de $\mathrm{n}=9$ a $\mathrm{n}=55$ e até $\mathrm{n}=58$.

Popescu et $a l .{ }^{27}$ mencionaram as vantagens no uso de um diodo termiônico como detector, demonstrando que este amplifica as cargas produzidas pelas ionizações direta e indireta resultantes do produto de fotoionização em vapores alcalinos, constituindo-se em um dispositivo útil para a técnica de espectroscopia de absorção. Devido à grande sensibilidade, esta técnica passou a ser importante em aplicações espectroscópicas envolvendo transições que ocorrem com baixa intensidade como, por ex., aquelas para altos estados excitados (desfavorecidas pelo pequeno valor dos momentos de transição) ou as que se originam de estados com pequena população. Assim, foram investigados espectroscopicamente íons formados ao incidir luz monocromática na descarga, sendo que as alterações na tensão foram registradas diretamente. Este novo tipo de espectroscopia passou a ser chamada de Espectroscopia de Impedância Óptica $^{34}$

Dentre as principais vantagens desta técnica em relação à espectroscopia de absorção convencional ou de fluorescência, pode ser citada a não necessidade do uso de fotodetetores e de fotomultiplicadoras, além da elevada eficiência na coleta do sinal. A Figura 4 mostra as principais técnicas de absorção em relação à espectroscopia de impedância óptica, mais conhecida, desde 1976 com o trabalho de Green et al..$^{22}$ em descargas elétricas $d c$ e em chamas, como espectroscopia optogalvânica.
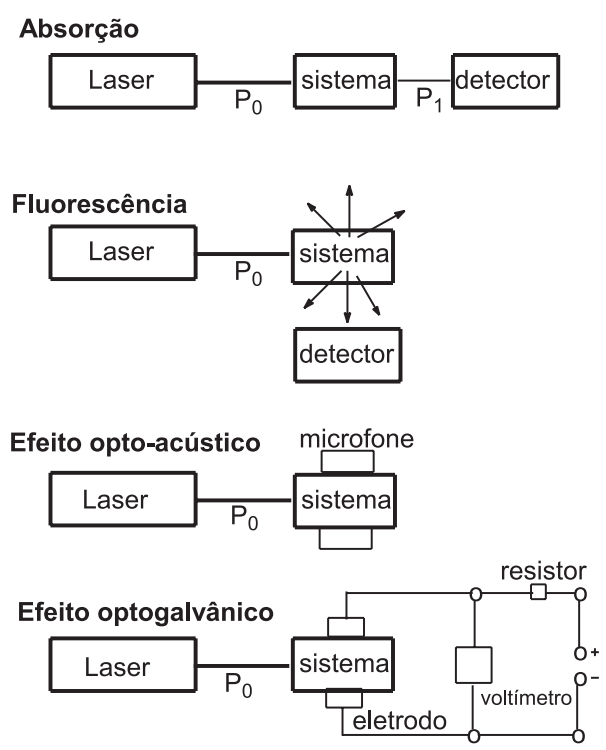

Figura 4. Esquema experimental das principais técnicas espectroscópicas, incluindo a espectroscopia optogalvânica

Com o desenvolvimento de lasers sintonizáveis de corante houve uma modernização das técnicas espectroscópicas de absorção e emissão. O primeiro trabalho envolvendo o uso deste equipamento na espectroscopia optogalvânica foi feito por Popescu et al. ${ }^{26}$, no qual foi identificada a formação do dímero $\mathrm{Cs}_{2}{ }^{+} \mathrm{em}$ uma descarga de vapor de césio, conforme mostram as Equações 3 e 4. Esta técnica possibilitou inúmeras vantagens para a espectroscopia multifotônica, sendo usada por Collins e colaboradores em processos de absorção de muitos fótons entre estados intermediários ${ }^{28}$, estados atômicos ${ }^{29}$ e estados dissociativos ${ }^{30,32,33}$.

$\mathrm{Cs}+2 \mathrm{hv} \rightarrow \mathrm{Cs}^{* *}+\mathrm{Cs}$

$\mathrm{Cs}^{* *}+\mathrm{Cs} \rightarrow \mathrm{Cs}_{2}^{+}+\mathrm{e}$

Portanto, como mostrado no trabalho de Popescu et al. ${ }^{29}$, o primeiro experimento sobre ionização com muitos fótons foi realizado em uma descarga de vapor de césio utilizando um laser de corante sintonizável que emite luz na região de 655-695 nm, sendo registrados os espectros de absorção de dois fótons.

\section{CONCLUSÕES}

Como apresentado neste trabalho, conclui-se que o efeito optogalvânico foi observado pela primeira vez por Foote e Mohler e, posteriormente, explicado por Penning em misturas de gases. Somente em 1937 Penning, através de uma patente ${ }^{40}$ demonstrou que este efeito também estava presente em gases ou vapores puros.

A utilização sistemática deste efeito como base para um novo tipo de espectroscopia deu-se, em 1964, com trabalhos de pesquisadores romenos que desenvolveram e aplicaram a técnica chamada por eles de espectroscopia de impedância óptica e denominada, a partir de 1976, de espectroscopia optogalvânica. 


\section{AGRADECIMENTOS}

Ao apoio financeiro recebido da CAPES e do CNPq.

\section{REFERÊNCIAS}

1. Goldsmith, J. E. M.; Lawler, J. E.; Contemp. Phys. 1981, 22, 235.

2. Barbieri, B.; Beverini, N.; Sasso, A.; Rev. Mod. Phys. 1990, 62, 603.

3. Foote, P. D.; Mohler, F. L.; Phys. Rev. 1925, 26, 195.

4. Mohler, F. L.; Foote, P. D ; Chenault, R. L.; Phys. Rev. 1926, $27,37$.

5. Penning, F. M.; Physica 1928, 8, 137.

6. Kenty, C.; Phys. Rev. 1950, 80, 95.

7. Meissner, K. W.; Miller, W. F.; Phys. Rev. 1953, 92, 896.

8. Phelps, A. V.; Molnar, J. P.; Phys. Rev. 1953, 89, 1202.

9. Garscadden, A.; Bletzinger, P.; Friar; E. M.; J. Appl. Phys. 1964, 35, 3432.

10. Freiberg, R. J.; Weaver, L. A.; J. Appl. Phys. 1967, 38, 250.

11. Carswell, A. I.; Wood J. I.; J. Appl. Phys. 1967, 38,3028.

12. Schenck, P. K.; Smyth, K. C.; J. Opt. Soc. Am. 1978, 68, 626.

13. Smyth, K. C.; Keller, R. A.; Crim, F. F.; Chem. Phys. Lett. 1978, 55, 473.

14. Erez, G.; Lavi, S.; Miron, E.; IEEE J. Quantum Electron. 1979, QE-15, 1328.

15. Lawler, J. E.; Phys. Rev. 1980, 28, 773.

16. Ferguson, A. I.; Phil. Royal Soc. Lond. A 1982, 307, 645.

17. Webster, C. R.; Rettner, C. T.; Laser Focus 1983, 19, 41.

18. Roesch, L.; Opt. Commun. 1983, 44, 259.

19. Doughty, D. K.; Lawler, J. E.; Appl. Phys. Lett. 1983, 42, 234.

20. Doughty, D. K.; Lawler, J. E.; Phys. Rev. 1983, 28, 773.

21. Rusak, D. A.; Anderson, J. E.; Keller, R. A.; Wilkerson, C. W.; Kunhardt, E. E.; Appl. Spectros. 1999, 53, 1651.

22. Green, R. B.; Keller, R. A.; Luther, G. G.; Travis, J. C.; Appl. Phys. Lett.
1976, 29, 727.

23. Badareu, E.; Popescu, I.; Chitã, C.; Zamfir, O.; Brit. J. Appl. Phys. 1964, $15,1171$.

24. Badareu, E.; Chitã, C.; Musa, G.; Popescu, I.;. Zamfir, O.; Brit. J. Appl. Phys. 1965, 16, 63.

25. Popescu, I.; Chitã, C.; Popescu, A.; Musa, G.; Ann. der Physik 1966, 18, 103.

26. Popescu, I.; Chitã, C.; Niculescu, N.; Phys. Lett. 1967, 24 A, 276

27. Popescu, D.; Pascu, M. L.; Collins, C. B.; Johnson, B. W.; Popescu I.; Phys. Rev. 1973, 8, 1666.

28. Collins, C. B.; Johnson, B. W.; Popescu, D.; Musa, G.; Pascu, M. L.; Popescu, I.; Phys. Rev. 1973, 8, 2197.

29. Popescu, D.; Collins, C. B.; Johnson, B. W.; Popescu, I.; Phys. Rev. 1974, 9, 1182.

30. Collins, C. B.; Johnson, B. W.; Popescu, D.; Popescu, I.; Phys. Rev. 1974 10,813 .

31. Popescu, D.; Popescu, I.; Maurer, J.; Collins, C. B.; Johnson, B. W.; Phys. Rev. 1975, 12, 1425 .

32. Collins, C. B.; Curry, S. M.; Johnson, B. W.; Mirza, M. Y.; Chellehmalzadeh, M. A.; Anderson, J. A.; Popescu D.; Popescu, I.; Phys. Rev. 1976, 14, 1662

33. Collins, C. B.; Anderson, J. A.; Lee, F. M.; Vicharelli, P. A.; Popescu, D.; Popescu I.; Phys. Rev. Lett. 1980, 44, 139.

34. Stanciulescu, C.; Bobulescu, R. C.; Surmeian, A.; Popescu, D.; Popescu I.; Collins, C. B.; Appl. Phys. Lett. 1980, 37, 888.

35. Langmuir, I.; Kingdon, K. H.; Science 1923, 12, 58.

36. Kingdon, K. H.; Langmuir, I.; Phys. Rev. 1923, 21, 380.

37. Penning, F. M.; Philos. Mag. 1929, 7, 632.

38. Penning, F. M.; Philos. Mag. 1931, 11, 961.

39. Penning, F. M.; U. S. Patente $n^{\circ} 1958066,1934$

40. Penning, F. M.; U. S. Patente $n^{\circ} 2080926,1937$. 